\title{
Determination of Antimicrobial Susceptibility Patterns between Different Strains of Propionibacterium Acnes
}

\author{
Yusufu, W.N. ${ }^{* 1}$, Suleiman, H. O. ${ }^{2}$, Akwa, V. Y. ${ }^{1}$, David, D. L. ${ }^{1}$, Taiga, A. ${ }^{1}$ \\ ${ }^{1}$ Department of Biological Sciences, Taraba State University Jalingo, Nigeria \\ ${ }^{2}$ Department of Pharmaceutics and Pharmaceutical Microbiology, University of Maiduguri, Nigeria \\ "Corresponding author: Yusufu,W.N.; waetsinya@gmail.com
}

Received 08 May 2019;

Accepted 13 June 2019;

Published 19 June 2019

\begin{abstract}
Propionibacterium acnes ( $P$. acnes) isolated in infections of deep tissues was originally thought to be as a result of improper sterilization of skin and hence contamination of medical processes with this commensal/ normal flora of the skin. $P$. acnes has latter been identified as the principal agent responsible for the progression of some deep tissue diseases. The antimicrobial susceptibility patterns between different strains of $P$. acnes was determined. Ten (10) P. acnes clinical isolates of infections were considered, two (2) from acne vulgaris and eight (8) [two (2) per recA types $1 \mathrm{~A} 1,1 \mathrm{~B}$, II and III] from lumber herniation tissues. Antibiotic susceptibility of some antibiotics was also determined and resistance to tetracycline was seen in 1 isolate, trimethoprim (1 isolate) and weak activity seen in 5 isolates. Heteroresistance to rifampicin was witnessed among 6 isolates. Upon four days of incubation, no inhibition of the bacterial growth by tetracycline in isolate 1(III). Isolate 17(1B) had no inhibition from trimethoprim. There was low inhibition of $P$. acnes by this antibiotic in isolates 71(IA1), 82(IB), 55(II), 1 (III) and 64(III). Amoxicillin and rifampicin created greater zones of inhibition among $P$. acnes isolates. Erythromycin was the next most inhibiting antibiotic with greater inhibition zones. Although Rifampicin had large inhibition zones, heteroresistance was observed among isolates 82(IB), 24(II), I (III), 17(IB), 55(II) and 71(IA1) as colonies were observed growing within the zones after 4 days of incubation. The antibiotics resistance expressed in the study were seen displayed among the lumber disc herniation isolates only with none involving acne vulgaris isolates. This study supports the theory that most $P$. acnes isolates in deep tissues exhibit higher rate of antibiotic resistance. $P$. acnes hence might be named the etiological agent of foreign-body infections like infections of indwelling medical devices.
\end{abstract}

Keywords: Propionibacterium acnes, Inhibition zones, Heteroresistance, Antibiotic susceptibility, Strains.

\section{Introduction}

Over a century ago, a patient suffering chronic skin disease "acne vulgaris" was the source of the initial Propionibacterium acnes ( $P$. acnes) isolate. Bacillus specie and then Corynebacterium species were the initial misclassification of the bacterium P. acnes (Bojar and Holland, 2004). Propionibacterium acne (P. acnes) is a slow growing, non-spore forming, aero tolerant, anaerobic, non-motile, Gram- positive, lipohilic, pleomorphic rod which belongs to the phylum Actinobacteria and class Propionibacteriales (Patrick and McDowell, 2012).

$P$. acnes is a primary inhabitant and colonizer of the human skin, conjunctiva, gut, intestinal tract and external ear canal along with Streptococcus, Corynebacterium, Staphylococcus and Pseudomonas species. P. acnes is usually not pathogenic (Cogen et al., 2008; Portillo, 2013).

P. acnes can be cultivated under anaerobic-to microaerophilic conditions using different culture media, (Patrick and McDowell, 2013) and a circular, opaque, about 1 to $2 \mathrm{~mm}$ in diameter and typically glistening are the characteristics of $P$. acnes colonies grown on blood agar plates. (Jousimies-Somer et al., 2002), growing better in anaerobic conditions but also possessing the ability to grow in aerobic conditions. The average detection time of $P$. acnes through blood cultures are about 6.4 days anaerobically and 6.1 days aerobically (Gunthard et al., 1994). Tissue cultures on the other hand needs more time for $P$. acnes to grow and therefore are incubated for 10 to 14 days (Schafer et al., 2008: Larson et al., 2012). For $P$. acnes to be considered an infections etiological agent, more than a single sample from blood culture has to read positive for $P$. acnes with the same isolate when distinguishing between a bloodstream infection and a skin contamination (Achermann et al., 2014).

Bruggemann et al., in 2004 was able to sequence the complete genome of $P$. acnes using the putative haemolysin and repair and maintenance (recA) of DNA genes. $P$. acnes strains from different infections were grouped into three types namely I, II and III using the whole genome sequencing (McDowell et al., 2008). This $P$. acnes recA gene DNA sequence analysis revealed the phylogenetically unique clusters/linage between the types I and II genotypes. The phylogenetic type III exhibited a difference in cell morphology and cell surface antigen (McDowell et al., 2008). 
These different types of $P$. acnes express different inflammatory properties and virulence factors (McDowell et al., 2005; Mc Dowell et al., 2008). A study by McDowell et al. (2008) used immunofluorescence microscopy and monoclonal antibodies (QUBPa1 and QUBPa2) which are precise to types I and II $P$. acnes to associate these two recA types with different infections. An approximately equal type I and type II strains population was recorded from detailed examination of isolates (P. acnes) found directly from prosthetic hip implants. Type I predominated isolates from related tissue and bone samples. Patient's surgical incision sites (skin), acne vulgaris and dental infection $P$. acnes isolates were found also to be predominated by type I. It was observed that the prosthetic hip implant had obvious type II isolates population (McDowell et al., 2008). Type III is associated with surgical excised spinal disc material (McDowell et al., 2008). The types IA and IB sub-strains within rec $A$ type I was identified using the $P$. acnes recA sequence analysis (Valanne et al., 2005). The whole genome sequencing and multilocus sequence typing (MLST) has been used to expand the above classification of $P$. acnes to types: $\mathrm{IA}_{1}, \mathrm{IA}_{2}, \mathrm{IB}, \mathrm{IC}, \mathrm{II}$ and III which the clinical and phenotypic levels of $P$. acnes back up (Mc Dowell et al., 2012).

The pathogenesis of the chronic skin infection acne vulgaris was thought to be associated directly to $P$. acnes but this has brought so much controversies in the science community due to its inability to satisfy Koch's postulate (Bojar and Holland, 2004: Segre, 2013).

As early as the 1980's, studies had suggested a relationship between the development of resistance by $P$. acnes to tetracycline, clindamycin, erythromycin and trimethoprim-sulfamethoxazole and the use of these antibiotics therapeutically (Eady et al., 1988: Eady et al., 1993: Cunliffe, 1995). The antibiotics resistance of $P$. acnes has been slowly rising from about $35 \%$ in 1991 to $60 \%$ in 1996 and this peaked up to 64\% in 1997 (Eady, 1998: Coates et al., 2002). Acne vulgaris is a chronic multifactorial disorder of the skin connected to a lot of pilosebaceous follicle factors and of the lipid rich sebaceous gland (Grange et al., 2009). Treatments of acne vulgaris acts through a mechanism of interference with the growth and metabolism of Propionibacterium (Oprica et al., 2004). A characteristics of acne vulgaris that questions $P$. acnes role as the disease's etiological agent is the incomplete response derived from the therapeutic options used which includes topical or systemic antimicrobial therapy to reduce bacteria load (Achermann et al., 2014). This antibiotic treatment failure has been related to the development of resistant strains in clinical isolates among patients (Leyden et al., 1983). This may be also due to biofilm intervening tolerance by bacteria (Ross et al., 2003). Analysis of some clinical trials carried out in the last two decades with the greatest evidence for the relationship between $P$. acnes and acne vulgaris showed decrease in the efficacy of erythromycin and clindamycin topical treatment in acne vulgaris. The production of resistant $P$. acnes strains was preceded by the decline in efficacy (Eady et al., 1989: Simonart and Dramaix, 2005). After treatment failure, recurrence of inflammation is witnessed supporting the theory of P. acnes not being the lone player in the pathogenicity of acne vulgaris since the specific antibiotic in therapeutic use might not be effective against other species of bacteria (Leyden, 2001).

\section{Materials and Methods}

Eleven (11) $P$. acnes isolates (10 clinical infection isolates and NCTC 737 a type I reference strain, \{biotype 3$\}$ ) were selected at random for this experiment, with no two (2) samples coming from the same source (patient). The sample size covered two (2) isolates per recA genotype $\left(1 \mathrm{~A}_{1}, 1 \mathrm{~B}\right.$, II, III). Lumber disc herniation provided eight (8) of the isolates while two (2) came from acne lesions. The used isolates were $71\left(1 \mathrm{~A}_{1}\right), 84\left(1 \mathrm{~A}_{1}\right), 17(\mathrm{IB}), 82(\mathrm{IB})$, 24(II), 55(II), 1(III), 64(III), acne lesion 1, acne lesion 7 and NCTC 737.

Chemicals/Reagents: Mueller- Hinton agar, Brain heart infusion agar and broth, purchased from Oxoid, United Kingdom. Antibiotics used were Ciprofloxacin $5 \mu \mathrm{g}$, Rifampicin $5 \mu \mathrm{g}$,

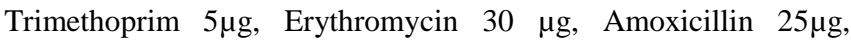

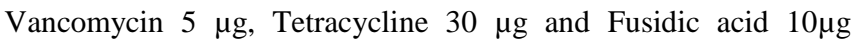
(Eucast 2016).

Materials: petri dishes, anaerobic incubator, spreader, centrifuge, Elisa auto reader (Tecan, sunrise plate reader, digital vernier caliper.

\section{Overnight cultures preparation}

Brain heart infusion agar (BHI) and broth (Oxoid) was prepared using the Manufacturer's prescription. Plates of BHI were used to anaerobically grow $P$. acnes cultures (by streaking) and incubated at $37^{\circ} \mathrm{C}$ for 4 days ( 96 hours). Overnight cultures were made from these pure $P$. acnes colonies by anaerobically growing them in sterile broth at $37^{\circ} \mathrm{C}$.

\section{Disc diffusion assay (EUCAST)}

Mueller-Hinton agar and Brain Heart infusion agar were inoculated with suspended $P$. acnes isolates from overnight culture standardised to an optical density of 0.1 at a wave length of 590nm with a colorimeter (Corning 253) by centrifugation and addition of sterile broth using the spread plate technique. The desired antibiotics disc was placed on the agar plates within $15 \mathrm{mins}$ of inoculation and incubated within 15mins. NCTC 737 was used as positive control. Plates were inverted and incubated anaerobically at $37^{\circ} \mathrm{C}$ for 4 days (96 hours). The diameter of zones of inhibition formed was measured to the nearest millimetre using a digital vernier calliper.

\section{Statistics}

Completely Randomized Design (CRD) as described by Gomez and Gomez (1984) was used with three replication and data obtained were analyzed statistically by Analysis of Variance (ANOVA) according to Gomez and Gomez (1984).'

\section{Result}

\section{Antibiotics disc diffusion assay}

After four (4) days of incubation, the result obtained showed that there was no inhibition of the bacterial growth by tetracycline in isolate 1(III). Isolate 17(1B) had no inhibition from trimethoprim. There was low inhibition of $P$. acnes by this antibiotic in isolates 71(IA1), 82(IB), 55(II), 1 (III) and 64(III). Greater zones of inhibition found among isolates of $P$. acnes were from the antibiotics amoxicillin and rifampicin. Erythromycin was the next most inhibiting antibiotic with greater inhibition zones (figure 1). Although Rifampicin had large inhibition zones, heteroresistance was observed among isolates 82(IB), 24(II), I (III), 17(IB), 55(II) and 71(IA1) as colonies were observed growing within the zones after 4 days of incubation. 


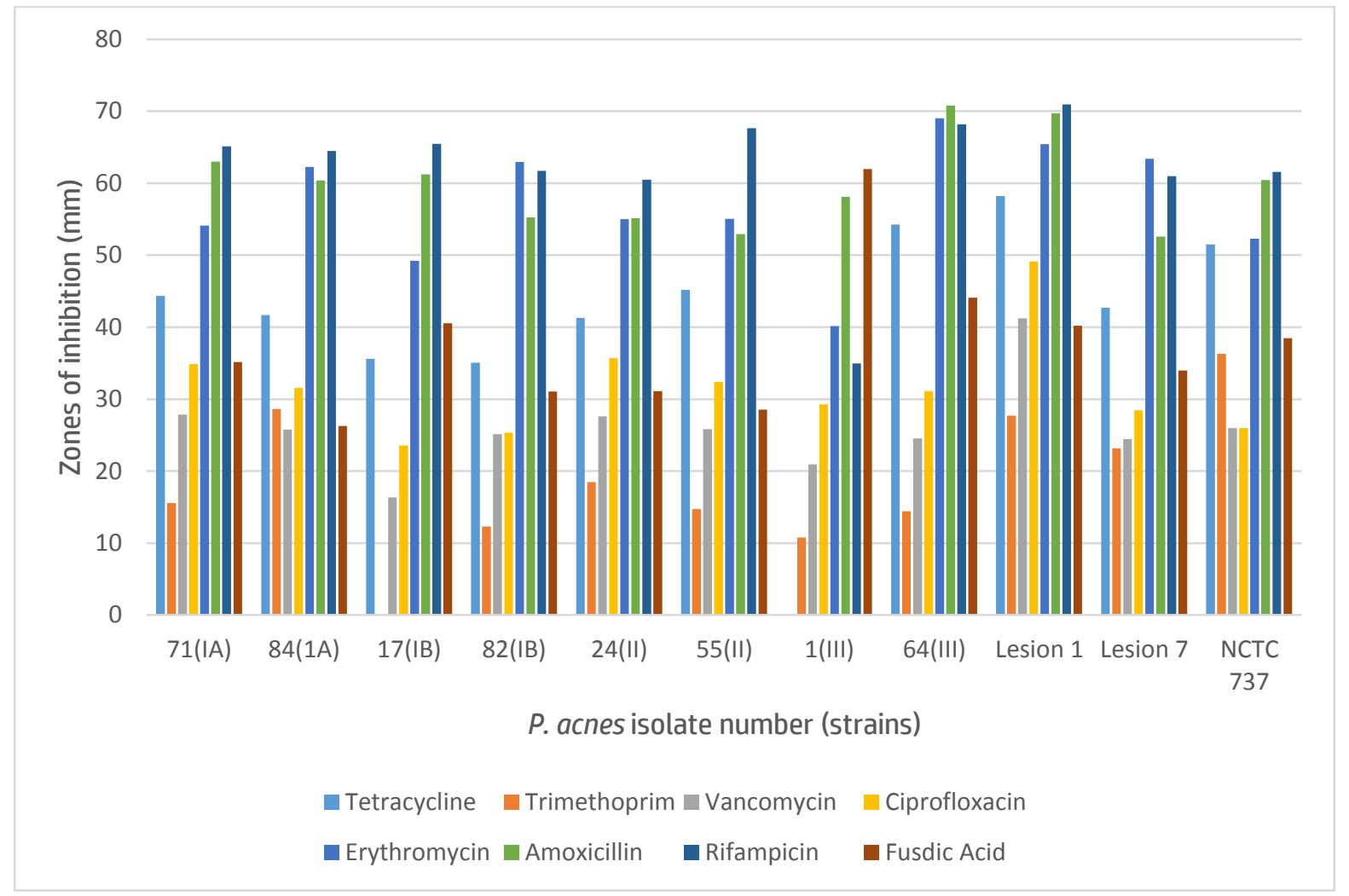

Figure 1: Zones of inhibition of different $P$. acnes isolates to a range of antibiotics on incubation for 4 days.

Table 1: Anova: Single Factor

\begin{tabular}{|c|c|c|c|c|c|c|}
\hline \multicolumn{7}{|l|}{ SUMMARY } \\
\hline Groups & Count & Sum & Average & Variance & & \\
\hline Row 1 & 8 & 340.02 & 42.5025 & 303.3514 & & \\
\hline Row 2 & 8 & 341.1 & 42.6375 & 292.6958 & & \\
\hline \multicolumn{7}{|l|}{ ANOVA } \\
\hline Source of Variation & $S S$ & $d f$ & $M S$ & $F$ & $P$-value & $F$ crit \\
\hline Between Groups & 0.0729 & 1 & 0.0729 & 0.000245 & 0.987742 & 4.60011 \\
\hline Within Groups & 4172.331 & 14 & 298.0236 & & & \\
\hline Total & 4172.403 & 15 & & & & \\
\hline
\end{tabular}

$\mathrm{P}$ is 0.988 which is greater than 0.05 this shows there is a significant difference in the effect of the different antibiotic treatments given to the samples.

\section{Discussion}

The statistical analysis used showed that there was a significant difference between the results obtained from the antibiotics used for this study. The study showed resistance in trimethoprim and tetracycline, which was only observed among lumber disc herniation and not an acne lesion isolates. These are commonly used antibiotics. Amoxicillin/ penicillin, clindamycin or linezolid/rifampicin are the most available current treatments for postsurgical or prosthetic P. acnes infections (Jakeb et al., 1996; Bayston et al., 2007: Nisbet et al., 2008). The role of antibiotics in the future therapy of back pain (Albert et al., 2008; Albert et al., 2013) was suggested by the isolation of $P$. acnes at the site of raptured disc as shown by Rollason et al., 2013. Although present back pain treatments focuses on controlling pain, physiotherapy and surgery (Rollason et al., 2013). Relating to the intensity of low back pain, a positive effect was observed in chronic back condition patients upon treatment with amoxicillin-clavulanate (Albert et al., 2008: Albert et al., 2013). This is similar to the result of this study, although amoxicillin was not used in combination, it exhibited a very high level of bacterial inhibition. The inhibition zones created by Amoxicillin can be questioned because it was 4 to 5 times more concentrated than the other antibiotics. The demonstration that anaerobic bacteria infected lumber disc have a higher possibility of developing modic changes and low grade discitis (Albert et al., 2013), and the evidence of the isolation of $P$. acnes from excised disc herniation tissue (Rollason et al., 2013) supports the theory of bacterial infection having an important role to play in this condition's development. Prolonged use of erythromycin and tetracycline for the treatment of acne vulgaris has led to the emergence of resistant $P$. acnes strains (Leyden et al., 1983; Eady et al., 1988; Kurokawa et al., 1999; Ross et al., 2003: Oprica et al., 2004). Although resistance to erythromycin was not witnessed in this study, which might be due to the fact that most of the clinical isolates used were from lumber disc herniation. Resistance to tetracycline was observed in one $P$. acnes isolate studied, which was also witnessed by Oprica et al. (2004). Resistance to trimethoprim was also observed and low/weak inhibition was seen in some isolates, this was also reported by Dessinioti and Dreno, 2015 and is similar to the findings of Oprica et al., 2004. Over the years, multiresistance has been observed to originate from isolates of acneic skin (McDowell et al., 2012). In contrast, all type IA1 isolates of $P$. acnes from disc tissues used in this study were sensitive to both erythromycin and tetracycline. 
Development of resistance by $P$. acnes to the antibiotic rifampicin has been reported by many researchers (Zappe et al., 2008; Furustrand et al., 2015). Furustrand, Trampuz and Corvec, 2013 described the molecular mechanism of resistance by rifampicin in $P$. acnes mutants which is due to point mutation in the $r p o B$ gene. Antimicrobial combinations which includes rifampicin is known to penetrate bacteria biofilms in Staphylococci implant associated infections and also has a positive effect on the bacteria in stationary phase (Zimmerli et al., 1998: Zimmerli et al., 2004). In 2012 , in vitro studies a foreign-body infection animal model were used to test the activity of rifampicin against $P$. acnes biofilms (Furustrand et al., 2012). Heteroresistance of $P$. acnes to antibiotics was also seen in this study in rifampicin with bacteria colonies growing in cleared zones although it was only observed in isolates from lumber disc herniation tissues only. There have been great studies done in the combination therapy of rifampicin with other antimicrobial agents to prevent emergence of rifampicin resistant strains due to monotherapy (Forrest and Tamura, 2010: Khassebaf et al., 2015). The inhibition zones created by rifampicin in the study can also be questioned because it was 4 to 5 times more concentrated than other antibiotics. The antibiotics resistance expressed in the study were seen displayed among the lumber disc herniation isolates only with none involving acne vulgaris isolates. The susceptibility of $P$. acnes planktonic cells is higher than sessile cells to antimicrobial agents and this has been reported (Ramage $e t$ al., 2003).

\section{Reference}

[1] Achermann, Y., Ellie, J. C., Coenye, T. and Mark, E. S. (2014). Propionibacterium acnes: from Commensal to Opportunistic Biofilm-associated Implant Pathogen. Clinical Microbiology Reviews, 27 (3): 419-440.

[2] Albert, H. B., Manniche, C., Sorensen, J. S., and Deleuran, B. W. (2008). Antibiotic treatment in patients with low-back pain associated with Modic changes type1 (bone oedema): a pilot study. British Journal of Sports Medicine, 42 (12): 969-973.

[3] Albert, H. B., Lambert, P., Rollason, J., Sorensen, J. S., Worthington, T., Pedersen, M. B., Norgaard, H. S., Vernallis, A., Busch, F., Manniche, C. and Elliott, T. (2013). Does nuclear tissue infected with bacteria following disc herniation's lead to Modic changes in the adjacent vertebrae? European Spine Journal, 22 (4): 690-696.

[4] Bayston, R., Nuradeen, B., Ashraf, W. and Freeman, B. J. C. (2007). Antibiotics for the eradication of Propionibacterium acnes biofilms in surgical infection. Journal of Antimicrobial Chemotherapy, 60 (6): 12981301.

[5] Bojar, R. A. and Holland, K. T. (2004). Acne and Propionibacterium acnes. Clinics in Dermatology, 22 (5): 375-379.

[6] Brüggemann, H., Henne, A., Hoster, F., Liesegang, H. (2004). The complete genome sequence of Propionibacterium acnes, a commensal of human skin. Science, 305: 671-673.

[7] Coates, P., Vyakrnam, S., Eady, E. A., Jones, C. E., Cove, J. H. and Cunliffe W. J. (2002). Prevalence of antibiotic-resistant Propionibacteria on the skin of acne patients: 10-year surveillance data and snapshot distribution study. British Journal of Dermatology, 146: 840-848.
[8] Cogen, A., L., Nizet, V. and Gallo, R. L. (2008). Skin microbiota: a source of disease or defence? British Journal of Dermatology, 158: 442-455.

[9] Cunliffe, W. (1995). Propionibacterium acnes resistance and its clinical relevance. Journal of Dermatological Treatment, 6 (1): S3-4.

[10] Dessinioti, C. and Dreno, B. (2015). Acne. European handbook of dermatological treatments. Edited by Andreas, K., Torello, L., Dessinioti, C. and Massimiliano D'Erme, A. Pages 7-17.

[11] Eady, E. A. (1998). Bacterial resistance in acne. Dermatology, 196: 59-66.

[12] Eady, E. A., Cove, J. H., Blake, J., Holland, K. T. and Cunliffe, W. J. (1988). Recalcitrant acne vulgaris, clinical, biochemical and microbiological investigation of patients not responding to antibiotic treatment. British Journal of Dermatology, 118: 415-423

[13] Eady, E. A., Cove, J. H., Holland, K. T. and Cunliffe, W. J. (1989). Erythromycin resistant Propionibacteria in antibiotic-treated acne patients: association with therapeutic failure. British Journal of Dermatology, 121 (1): 51-57.

[14] Eady, E. A., Jones, C. E., Tipper, J. L., Cove, J. H., Cunliffe, W. J. and Layton A. M. (1993). Antibiotics resistant Propionibacteria in acne: need for policies to modify antibiotics usage. British Medical Journal, 306: 555-556

[15] Eucast (2016). Eucast disc diffusion methodology [online] available from <http://www.eucast.org/ast_of_bacteria/disk_diffusion_ methodology/> [12 August 2016].

[16] Forrest, G. N. and Tamura, K. (2010). Rifampin combination therapy for non-mycobacterial infections. Clinical Microbiology Reviews 23 (1): 14-34.

[17] Furustrand, T. U., Aubin, G. G., Eich, G., Trampuz, A. and Corvec, S. (2015). Occurrence and new mutations involved in rifampicin- resistant Propionibacterium acnes strains isolated from biofilm or device-related infections. Anaerobe, 18: 116-119.

[18] Furustrand, T. U., Corvec, S., Betrisey, B., Zimmerli, W. Trampuz A. (2012). Role of rifampicin against Propionibacterium acnes biofilm in vitro and in an experimental foreign-body infection model. Antimicrobial Agents Chemotherapy, 56 (4): 1885-1891

[19] Furustrand, T. U., Trampuz, A. and Corvec, S. (2013). In vitro emergence of rifampicin resistance in Propionibacterium acnes and molecular characterization of mutations in the rpoB gene', Journal of Antimicrobial Chemotherapy, 68 (3): 523-528

[20] Grange, P.A., Chereau, C., Raingeaud, J., Nicco, C., Weill, B., Dupin, N. and Batteux, F. (2009). Production of superoxide anions by keratinocytes initiates $P$. acnesinduced inflammation of the skin'. PLoS Pathogens, (5): e1000527.

[21] Gomez, K. A. and Gomez A. A. (1984). Statistical procedures for Agricultural research. 2nd Edition, John Wiley and sons, New York, Pp 680.

[22] Gunthard, H., Hany, A., Turina, M. and Wust, J. (1994). Propionibacterium acnes as a cause of aggressive aortic valve endocarditis and importance of tissue grinding. Case report and review. Journal of Clinical Microbiology, 32: 3043-3045. 
[23] Jakab, E., Zbinden, R., Gubler, J., Ruef, C., von Graevenitz, A. and Krause, M. (1996). Severe infections caused by Propionibacterium acnes: an underestimated pathogen in late postoperative infections. Yale Journal of Biology and Medicine, 69 (6): 477-482.

[24] Jousimies-Somer, H., Summanen, P., Citron, D., Baron, E., Wexler, W., Finegold S. (2002). Wadsworth-KTL anaerobic bacteriology manual. 6th ed. Star Publishing Co, Belmont, CA

[25] Khassebaf, J., Hellmark, B., Davidsson, S. and Unemo, M. (2005). Antibiotic susceptibility to Propionibacterium acnes isolated from orthopaedic implant-associated infections. Anaerobe, 32: 57-62.

[26] Kurokawa, I., Nishijima, S. and Kawabata, S. (1999). Antimicrobial susceptibility of Propionibacterium acnes isolated from acne vulgaris. European Journal of Dermatology, 9: 25-28.

[27] Larson, L. H., Lange, J., Xu, Y. and Schonheyder, H. C. (2012). Optimizing culture methods for diagnosis of prosthetic joint infections: a summary of modifications and improvements reported since 1995. Journal of Medical Microbiology, 61: 309-316.

[28] Leyden, J. J. (2001). Current issues in antimicrobial therapy for the treatment of acne. Journal of European Academy of Dermatology and Venereology, 15(3): 5155.

[29] Leyden, J. J., McGinley, K. J., Cavalieri, S., Webster, G. F., Mills, O. H. and Kligman, A. M. (1983). Propionibacterium acnes resistance to antibiotics in acne patients. Journal of American Academy of Dermatology, 8: $41-45$

[30] McDowell, A., Barnard, E., Nagy, I., Goa, A. Tomida S, Li H, Eady A, Cove J, Nord CE, Patrick S. (2012). An expanded multilocus sequence typing scheme for Propionibacterium acnes: investigation of "pathogenic", "commensal" and antibiotic resistant strains. PLOS ONE 7 (7): e41480.

[31] McDowell, A., Perry, A. L., Lambert, P. A. and Patrick, S. (2008). A new phylogenetic group of Propionibacterium acnes. Journal of Medical Microbiology (57): 218-224.

[32] McDowell, A., Valanne, S., Ramage, G., Tunney, M. M., Glenn, J. V., McLorinan, G. C., Bhatia, A., Maisonneuve, J. F., Lodes, M., Persing, D. H. and Patrick, S. (2005). Propionibacterium acnes types I and II represent phylogenetically distinct groups. Journal of Clinical Microbiology (43): 326-334.

[33] Nisbet, M., Briggs, S., Ellis-Pegler, R., Thomas, M. and Holland, D. (2007). Propionibacterium acnes: an underappreciated cause of post neurosurgical infection. Journal of Antimicrobial Chemotherapy, 60 (5): 10971103.

[34] Oprica, C., Emtestam, L., Lapins, J., Borglund, E., Nyberg, F. Stenlund K, Lundeberg L, Sillerström E, Nord CE. (2004). Antibiotics resistant Propionibacterium acnes on the skin of patients with moderate to severe acne in Stockholm. Anaerobe, 10: 155-64.

[35] Patrick, S. and McDowell, A. (2012). "Propionibacterium". In: Goodfellow, M., Kampfer, P., Busse, H. J., Trujillo, M. et al. editors. The
Actinobacteria Bergy's Manual of systematic bacteriology $5,2^{\text {nd }}$ edition New York: Springer.

[36] Patrick, S. and McDowell, A. (2013). Propionibacterium acnes: an emerging pathogen in biomaterial-associated infection. In Moriarty TF (ed), Biomaterials associated infection: immunological aspects and antimicrobial strategies. Springer Science and Business Media, New York, NY.p 87-105

[37] Portillo, M. E., Corvec, S., Borens, O. and Trampuz, A. (2013). Propionibacterium acnes: an underestimated pathogen in implant-associated infections. Biomed Research International, Article ID 804391

[38] Ramage, G., Tunney, M. M., Patrick, S., Gorman, S. P. and Nixon, J. R. (2003). Formation of Propionibacterium acnes biofilms on orthopaedic biomaterials and their susceptibility to antimicrobials. Biomaterials 24, 32213227.

[39] Rollason, J., Dowell, A., Albert, H. B., Barnard, E., Tony W., Anthony, C. H., Ann, V., Sheila, P., Tom, E. and Peter, L. (2013). Genotypic and Antimicrobial Characterisation of Propionibacterium acnes Isolates from Surgically Excised Lumbar Disc Herniations. BioMed Research International, 530382, 1-7.

[40] Ross, J. I., Snelling, A. M., Eady, E. A., Cove, J. H., Cunliffe, W. J., Leyden, J. J., Collignon, P., Dreno, B., Reynaud, A., Fluhr, J. and Oshima, S. (2001). Phenotypic and genotypic characterization of antibioticresistant Propionibacterium acnes isolated from acne patients attending dermatology clinics in Europe, the U.S.A., Japan and Australia. British Journal of Dermatology, 144:339-346.

[41] Schafer, P., Fink, B., Sandow, D., Margull, A., Berger, I. and Frommelt, L. (2008). Prolonged bacterial culture to identify late periprosthetic joint infection: a promising strategy. Clinical Infectious Diseases, 47:1403-1409.

[42] Segre, J. A. (2013). What does it take to satisfy Koch's postulates two centuries later? Microbial genomics and Propionibacterium acnes. Journal of Investigative Dermatology, 133: 2141-2142.

[43] Simonart, T. and Dramaix, M. (2005). Treatment of acne with topical antibiotics: lessons from clinical studies. British Journal of Dermatology 153: 395-403.

[44] Valanne, S., McDowell, A., Ramage, G., Tunney, M. M., Einarsson, G. G., O’Hagan, S., Wisdom, G. B., Fairley, D., Bhatia, A., Maisonneuve, J. F., Lodes, M., Persing, D. H. and Patrick, S. (2005). CAMP factor homologues in Propionibacterium acnes: a new protein family differentially expressed by types I and II. Microbiology, (151): 1369-1379

[45] Zappe, B., Graf, S., Ochsner, P.E., Zimmerli, W. and Sendi P. (2008). Propionibacterium spp. in prosthetic joint infections: a diagnostic challenge. Archives Orthopaedic and Trauma Surgery, 128 (10):1039-1046.

[46] Zimmerli, W., Trampuz, A. and Ochsner, P. E. (2004). Prosthetic-joint infections. New England Journal of Medicine, 351 (16): 1645-1654.

[47] Zimmerli, Z., Widmer, A.F., Blatter, M., Frei, R. and Ochsner, P. E. (1998). Role of rifampicin for treatment of orthopaedic implant-related staphylococcal infections: a randomized controlled trial. Foreign-Body Infection (FBI) Study Group, JAMA 279 (19): 1537-1541. 